Sintesis:

Este trabajo surge a partir de la investigación sobre los discursos generada por el Departamento de Lenguaje y Comunicación de la Facultad de Ciencias de la Comunicación. Durante ese proceso la temática se abre en dos vertientes: una, interesada en los denominados discursos mediáticos y otra que indaga sobre los usos discursivos manifestados en el ámbito estudiantil.

El punto de partida vincula aspectos de lectura y escritura con el uso lingúístico, profundamente marcado por las variaciones semióticas provenientes del contexto, en el cual existe una constante acción de los medios, cuya influencia en los modelos mentales de los jóvenes puede inferirse a partir de los resultados que arrojan los itemes de la encuesta que acompaña la investigación, algunos de los cuales se presentan en este artículo.

Entre las constataciones más importantes se verifica la presencia preponderante de la televisión en el ámbito en el cual los jóvenes interactủan y estudian; en tanto que las denominadas nuevas tecnologias de la información amplian

"su espacio y presencia. Enfrentada a estos hechos la universidad tiene un papel preponderante en relación con la responsabilidad social que acompaña su labor educativa.

Palabras clave: contexto, interpretante, modelo mental, neogramática, transcripción semiótica, variación semiótica.

Synthesis

This work happens as from the investigation on the discourses generated by the Department of Language and Communication from the Communication's Science Faculty. During the process the subject matter diverges in two ways: one, interested in the named media discourses and another one that investigates the discursive uses manifested in the student space.

The starting point links aspects of reading and writing with the linguistic use, deeply mark by the semiotic variations that come from the context, that exists in a constant action of the means, whose influence in young people's mental models can be inferred as from the results of the opinion poll that is annexed

to the investigation, some are presented in this article.

Among the most important verifications, it is verified the preponderant presence of the television in the space which young people interact and study with; while the denominated information's new technologies increases their space and presence. Confronted with this facts the university has a preponderant roll related to the social responsibility that comes with the educational work.

Key Words: Context interpretant, mental model, neogramática, transcription semiotics, variation semiotics,

\title{
Los estudiantes
}

universitarios y los discursos.

\section{La posibilidad de una neogramática.}

María Cristina Asqueta Corbellini

Docente e investigadora de la Facultad de Ciencias de la Comunicación de

Uniminuto, en 2007 se ha desempeñado como directora de la Especialización en Comunicación Educativa. Adelanta estudios de doctorado en Vanguardias y postvanguardias en España e Hispanoamérica, de la Universidad de Salamanca

(España).

e-mail: masqueta@uniminuto.edu

Clarena Muñoz Dagua

Docente e investigadora de la Universidad Colegio Mayor de Cundinamarca. Actualmente, estudia el doctorado en Análisis del Discurso en la Universidad de Buenos Aires (Argentina). e-mail: clargui@yahoo.es

Miriam Casco Docente e investigadora de la Universidad Nacional del Centro de la Provincia de Buenos Aires. Magister en Lingüistica Española del Instituto Caro y Cuervo

(Colombia).

e-mail. mcasco@arnet.com.ar

\section{Introducción}

\section{La reflexión que aquí se presenta surge de la investigación denominada: La neogramática del estudiante universitario, realizada mediante convenio suscrito}


por tres universidades: Universidad Colegio Mayor de Cundinamarca; UNIMINUTO Corporación Universitaria, de Colombia; Universidad Nacional del Centro de Buenos Aires, de Argentina. La investigación, planteada a partir de los procesos de investigación y de docencia liderados por el Departamento de Lenguaje y Comunicación de la Facultad de Ciencias de la Comunicación, se desarrolló durante 2005 y 2006 con la elaboración del marco teórico, el diseño y aplicación de encuestas entre los estudiantes de primer semestre, en diferentes carreras.

La pregunta inicial con la cual se origina la investigación vincula aspectos de lectura y escritura con el uso lingüístico, profundamente marcado por las variaciones semióticas (Klinkenberg, 2006) características del uso lingǘstico influenciado por aspectos espaciales,

" temporales y sociales y, del mismo modo, de los estudiantes que ingresan a la educación superior. La universidad constituye un espacio abierto en el cual se produce un choque cultural al confrontar el modelo educativo con los ingresantes, en cuanto son personas formadas en contextos donde interactúan con distintos modelos tanto lingüísticos y comunicativos como cognitivos. El punto de vista acerca de cómo debe ser la educación superior, a pesar de las reflexiones y argumentos, en apariencia sólidos, si no está basada en una flexibilidad la cual, sin descuidar la calidad académica, comprende también la variación, se transforma en un obstáculo para el cumplimiento de la vida académica y en un fracaso de la educación en cuanto tiene que ver con la formación integral. En el caso colombiano, se puede detectar este problema con la interpretación de los resultados de las pruebas de Estado ECAES, cuyo componente denominado expresión ha obtenido porcentajes muy bajos para producir, como podria esperarse, alarma entre los académicos.
Los usos lingüísticos adquiridos por los jóvenes en su cultura afectan los logros académicos tanto como influyen los prejuicios, preconceptos y concepciones que conforman los modelos mentales' (cognoscitivos) desde los cuales ellos abarcan los contextos en los que interactúan.

Las investigadoras no están alineadas con posturas dogmáticas o conservacionistas, aferradas a determinada norma o uso denominado culto; muy al contrario, tienen claridad sobre la necesidad de que el ámbito universitario esté preparado para interactuar con la variación y, por ende, existan condiciones para afectarla o adecuarla, de acuerdo con las habilidades en las cuales se forman los profesionales, destinados a desarrollar acciones relevantes para el conjunto social. Además, se considera la incidencia de las llamadas nuevas tecnologías de la información (TiC) en los hábitos de los estudiantes como parte del reto planteado por estos contextos?

Las TIC cuentan con expertos entre los jóvenes estudiantes pero con ellas, a la inversa, se vinculan con menor fluidez los estamentos académicos donde aún el libro, el papel y el tablero constituyen el instrumento y soporte de las comunicaciones educativas; entonces, a pesar del significativo avance del video-been y del computador personal utilizados en las presentaciones power-point, la clase consiste en leer en la pantalla

\footnotetext{
'El concepto de modelos mentales pertenece a Teun Van Dijk en Discurso y dominación, en Grandes conferencias en la Facultad de Ciencias Humanas No. 4, febrero de 2004, para quien en la conformación de estos modelos intervienen los mecanismos de control de las elites simbólicas, ente las cuales ubica a los medios de comunicación, con los cuales las personas son dominadas. ${ }^{2}$ Contexto refiere acá a modelos cognitivos para interpretar, actuar y comprender el mundo, de acuerdo con los avances planteados por Teun van Dijk en su conferencia Discurso y dominación.
} 


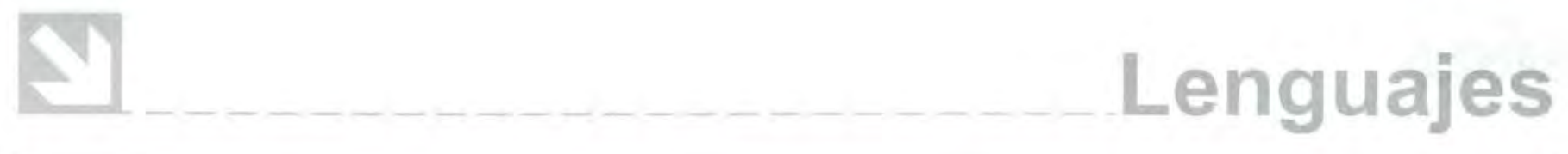

12

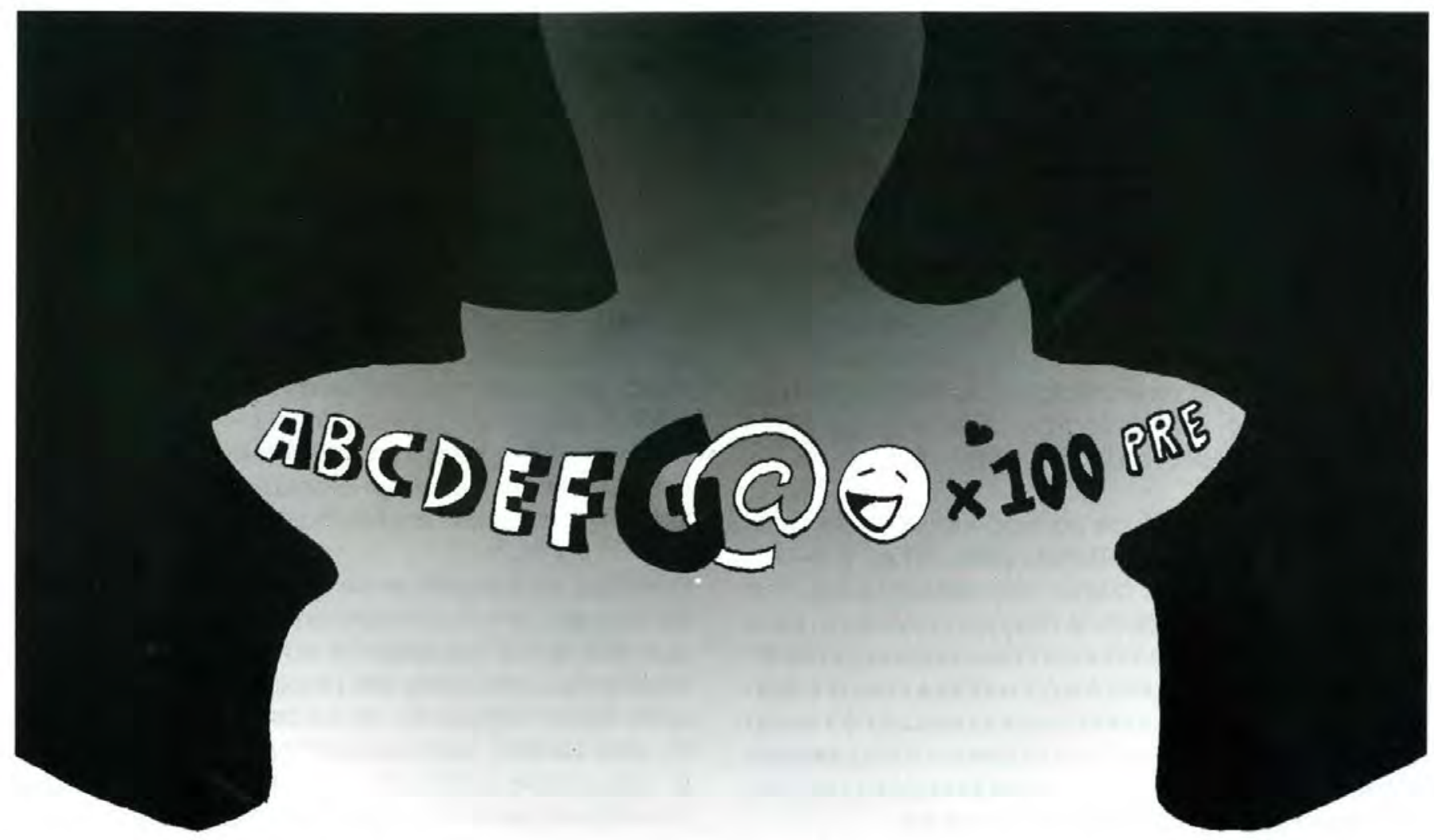

bidimensional cuando, en la práctica anterior, el docente escribía sus explicaciones directamente en el tablero, ayudado por un significativo ejercicio corporal en la dińámica de la clase.

El término gramática, del cual se parte, posee doble significación. Por un lado, refiere una disciplina nacida en la antigüedad griega, siglo $V$ a. C., como parte de la Filosofía y, por el otro, designa usos mediante los cuales operan los códigos.
Ambos aspectos constituyen una asociación porque la gramática se considera una creación cultural, de ahí la arbitrariedad que la caracteriza y que la hace estar fuertemente vinculada al poder, por lo cual conforma también una imposición y, como material de uso, resulta vulnerada por las variaciones. A partir de estas características es posible pensar la neogramática como el aspecto contemporáneo del código lingüístico y de los demás códigos, ya que como lo propone Klinkenberg (2006, 219-224) en la comunicación no opera un único código sino que se trata de pluricódigos. 


\section{La complejidad de la relación entre el código lingüístico y el uso}

La concepción de la variabilidad ha sido estudiada por la Lingüística, disciplina escindida de la gramática que supera los aspectos dogmáticos al comprender aspectos cognoscitivos y pragmáticos. En el inicio de su trabajo sobre el signo lingüístico, F. de Saussure preveía la mutalidad nombre con el cual en el Curso de lingüística general (Saussure, 1945) se designaba la variación; a partir de sus postulados, las ramas de la clencia del lenguaje como la dialectología y la sociolingüística, principalmente, se dedicaron al estudio de estos cambios temporales, espaciales y sociales.

El concepto de variación ha sido incorporado a los estudios de la semiótica (Klinkenberg, 2006, 119-179) al describir los códigos, cuyas características provienen de estas dinámicas. Además, hay que considerar queel contexto contemporáneo y los camblos causados por las TIC crean mayor complejidad en las comunicaciones yenfatizan la mutabilidad. En consecuencia, se produce la confrontación con aquellos espacios socio-comunicativosen los cuales existe latendencia a la conservación, con predominio de posiciones fundamentadas en la tradición y en los hábitos.

El mencionado principio de la mutabilidad, postulado por Saussure, consiste sólo en un aspecto del código verbal el cual es complementado por una oposición. denominada por el lingüista inmutabilidad, la cual no es otra cosa que la defensa del sistema para no desintegrarse con los cambios y garantizar la comunicación, que depende de la redundancia, la revisión y la confirmación de los usos.

Instrumentos como messenger y chat, cuando se utilizan como medio escrito, permiten ver cómo los denominados emoticones, signos iconográficos no verbales, integran una estructura discursiva que surge de una nueva escritura jeroglífica-ideográfica en movimiento, ésta parece desplazar a la avanzada escritura fonológica que la sustituye durante la historia de la cultura.

Esta nueva escritura comúnmente se denomina Icono porque representa ideográficamente un objeto o emoción. En este uso se verifica también lo que Jean-Marie Klinkenberg (2006) denomina transcripción semiótica cuando existe un determinado código interpretado por otro; por ejemplo, el código sonoro complementado o sustituido por el código visual. También se constata transcripción entre códigos que usan un mismo canal, un procedimiento habitual de las comunicaciones contemporáneas.

Entonces, el contraste entre variación y conservación de los usos produce confrontaciones y conflictos que pueden encauzarse al adquirir las instituciones educativas conciencia del problema y, sobre todo, a partir de la investigación de los aspectos involucrados. En este sentido, dado el compromiso formativo de la universidad están en juego las habilidades que canalizan el desarrollo de las personas y los grupos, con miras al bienestar individual y social.

\section{El lenguaje de la exclusión}

Como manifestación del problema, en la educación superior, a pesar del avance del despliegue tecnológico, es posible advertir en clase a estudiantes que duermen, conversan entre ellos e ignoran qué sucede en el espacio docente con notoria ausencia de interacciones maestro-alumno. Los estudiantes marginados existen en gran número, sin que se hayan modificado las dificultades cognoscitivas detectadas hace diez, veinte $y$ treinta años. Se ha reconstruido la fachada $y$ 


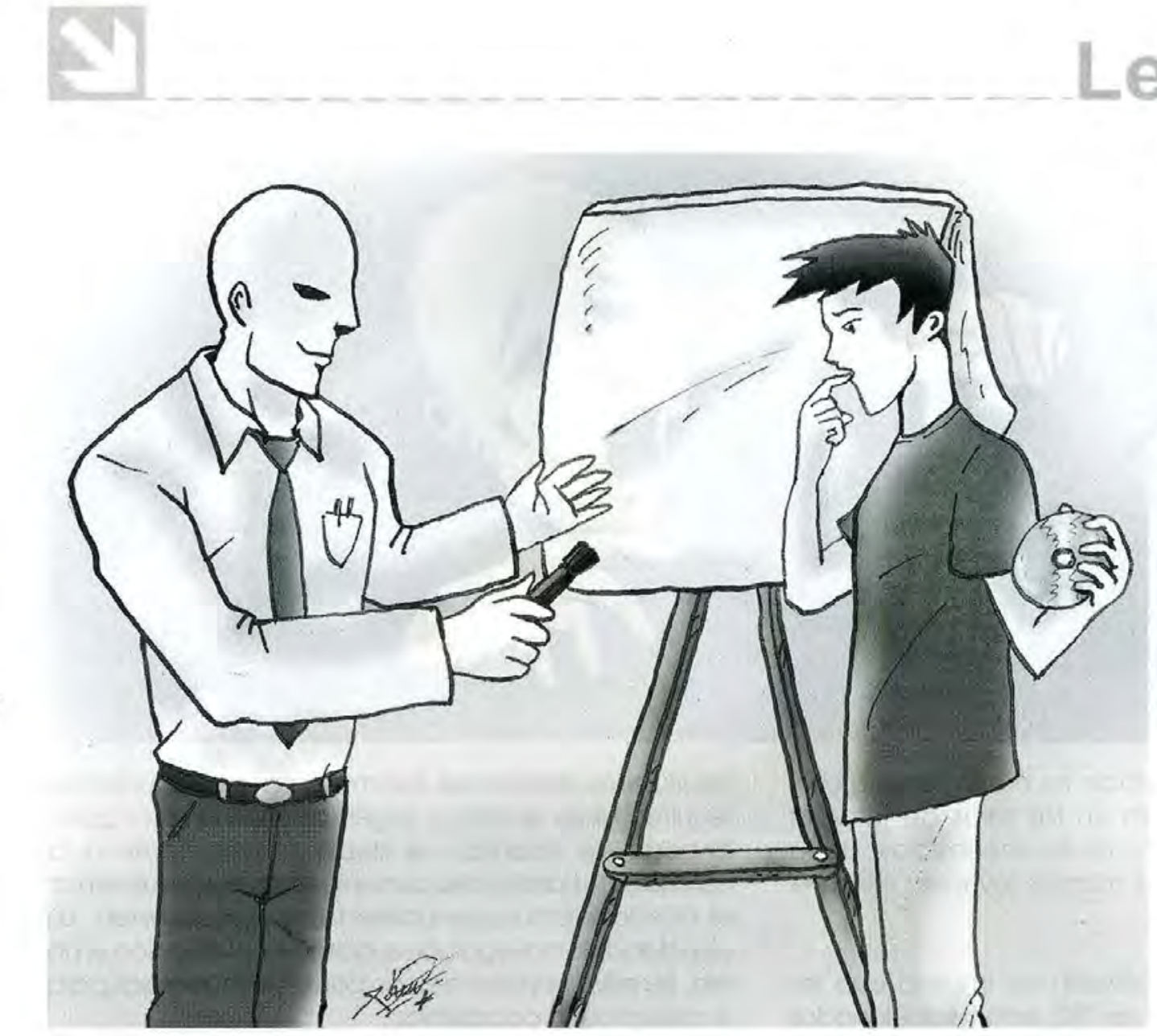

\section{Enredados con la red}

Además, es necesario considerar que no todo el ámbito universitario está integrado exclusivamente por las élites que disponen de la tecnología incluso de computador en su casa, o en el colegio, característica permanente que acompaña al subdesarrollo, el cual convive con los avances tecnológicos, aunque la educación pública ha mejorado sensiblementey también las posibilidades de la población estudiantil de acercamiento a dichos recursos. Cito como ejemplo el de los colegios distritales de Bogotá que como lo

hasta el interior, pero aún existen numerosos excluidos del proceso formativo universitario y muchos lo son porque permanecen mudos, inseguros, tímidos y sin retroalimentación; aunque hábiles navegadores, muchas veces, no revierten esa información, más allá del plagio, en los procesos evaluativos.

consigna la especialista en comunicación educativa Sandra Patricia Garzón (2007) en su trabajo Criterios educomunicativos para la creación de un sitio web como herramienta pedagógica ${ }^{3}$ los alumnos acceden a las tecnologías en los colegios y las bibliotecas

${ }^{3}$ Trabajo de grado presentado por la autora para obtener su título de Especialista en Comunicación Educativa, en la Facultad de Ciencias de la Comunicación de UNIMINUTO, Corporación Universitaria 


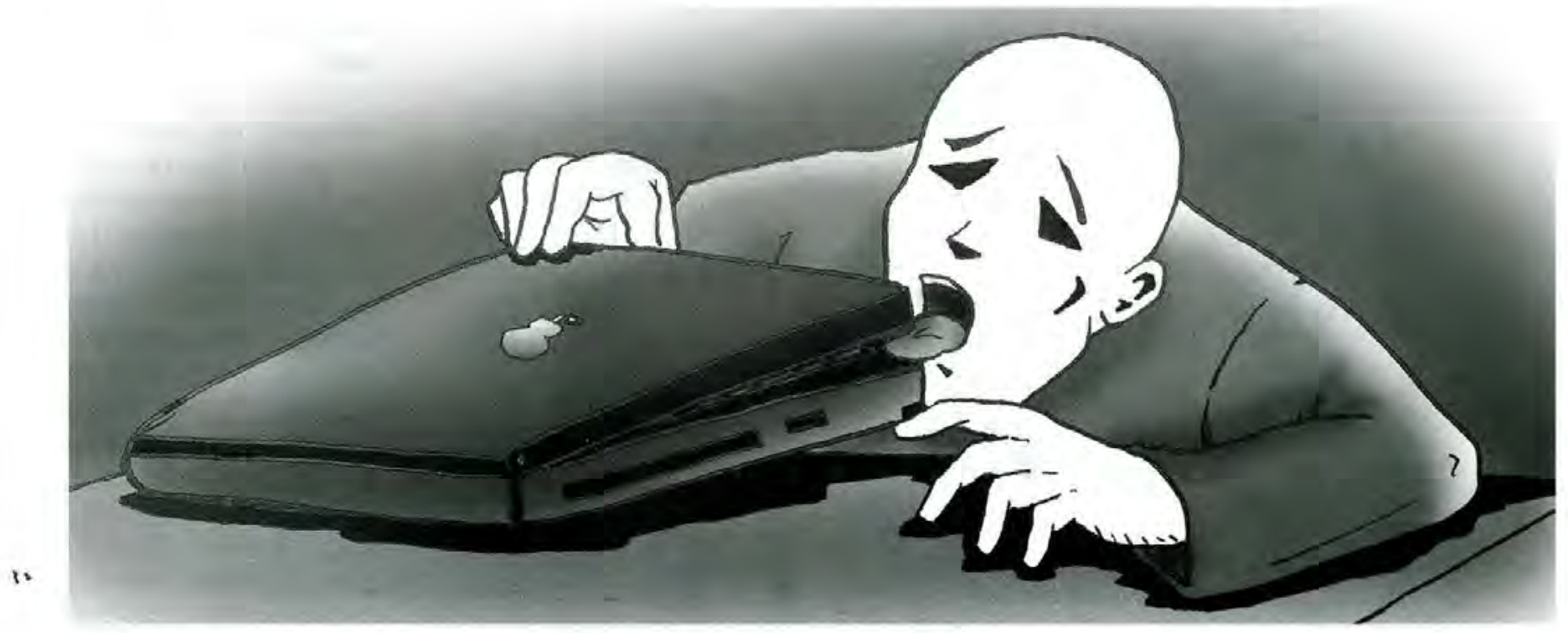

Ios cuales han incrementado su oferta de material interactivo; pero también en los sitios de Internet presentes en sus barrios a costos accesibles, de la misma manera que estos mismos jóvenes utilizan el teléfono celular.

Sin embargo, esta constatación no implica que los problemas de acceso a las TIC estén solucionados y mucho menos su participación en el desarrollo del conocimiento en una comunidad educativa; ello no impide referenciar el avance avasallador de los contextos tecnológicos los cuales sin duda representan ventajas para la educación y, a la vez, comprenden un número significativo entre la población joven en formación en los colegios y con posibilidades de ingresar a la educación superior.

La universidad aún los recibe con un aula básicamente oral en la cual también falta interacción. En cuanto a la

escritura las dificultades son mayores; si los estudiantes recurren tanto al plagio, según puede verse en cada entrega de trabajos, se debe precisamente a la carencia de habilidades comunicativas para representar el conocimiento que poseen o que adquieren. La velocidad de navegación e incluso la interacción en la red, en estas condiciones, no constituyen ventajas para el desempeño académico.

La red conforma un diccionario y una enciclopedia ${ }^{4}$; con los cuales podría consolidarse el proceso educativo universitario. Sin embargo, desde la red llegan las páginas simplemente copiadas y sin transformaciones, a manos de docentes, para que los evalúen como elaboración

${ }^{4}$ Estos términos provenientes de la semiótica peirceana constituyen un argumento fundamental en el concepto de interpretante, muchas veces ausente en el aula con los consiguientes perjuicios para la labor educativa. 
personal; entonces surgen los conflictos entre profesores y estudiantes. La comunidad educativa también se afecta cuando advierte que los jóvenes permanecen en la red en actividades de comunicación: messenger, chat, correo electrónico y con amplio acceso a la oferta publicitaria, en tanto fetiche mercantil ${ }^{5}$, incluso pornografía, sin que apropien los beneficios del conocimiento que aunque más escaso circula por la misma red. Vale la pena recordar que estos recursos también están vinculados al consumismo.

Por tanto, a partir de estas influencias se infiere la existencia de una nueva gramática en disputa en los escenarios del ámbito contemporáneo. Esta neogramática está disponible para la comunicación y posiblemente la enriquece pero, por ahora, también da lugar a nuevas marginaciones y escasamente genera aptitudes y habilidades para el desarrollo del pensamiento que, en la universidad, representa el desarrollo científico.

\section{Antecedentes teóricos}

Esta investigación cuenta, entre sus precedentes, con el trabajo del Profesor Fabio Jurado Valencia sobre la escritura como proceso semiótico reestructurador de la conciencia elaborado a partir de investigaciones en el ámbito de la academia universitaria, una labor vinculada con las reformas educativa adelantadas en la Univérsidad Nacional de Colombia a partir de la necesidad de desarrollar la academia sobre la base de lecturas analíticas y consecuentes recontextualizaciones teóricas con la propuesta de practicar en las aulas la exposición oral, apoyada en la exposición escrita para asegurar la cohesión de los procesos cognitivos

${ }^{5}$ Este concepto se aplica en el sentido de la Escuela Crítica, de Th. Adorno y W. Benjamin sobre la sociedad del consumo. y dinamizar la amergencia del sujeto epistémico, necesario en la academia universitaria.

También, se encuentran entre las fuentes las investigaciones sobre lectura y escritura en la educación superior de la Universidad de Medellin, con la orientación de la coordinadora general de la Cátedra UNESCO para la Lectura y la Escritura en América Latina, María Cristina Martínez.

Los trabajos mencionados pertenecen al ámbito latinoamericano pero en el orden internacional son reconocidos los estudios e influencia de Danlel Cassany. También, las organizaciones que hacen un esfuerzo en torno a la alfabetización como International Reading Asociation, IRA, que permanentemente abren espacios a la investigación y reflexión de estos temas.

\section{El discurso de los universitarios}

La investigaclón sobre la neogramática toma en consideración los discursos de los estudiantes cuando ingresan a la universidad. En ese sentido se efectúa la descripción del ámbito en el cual se produce el problema, elaborada en una parte con base en la experiencia de las investigadoras, quienes desempeñan su labor docente y de investigación en las áreas de lenguaje de diferentes carreras de las tres universidades. Esta reconstrucción contextual se complementa con los argumentos teóricos que sustentan el trabajo, aportados por la teoría lingüística, el análisis del discurso y la semiótica. En síntesis, el sello propio del trabajo proviene de la semiolingüística, al indagar en representaciones que no son sólo verbales-auditivas sino también verbalesvisuales (escrituras) o estrictamente visuales (iconografias). La investigación, empero, se amplía con la aplicación de encuestas de las cuales surgen unas estadísticas que sometidas a la interpretación muestran algunas 


\section{Irequinciones}

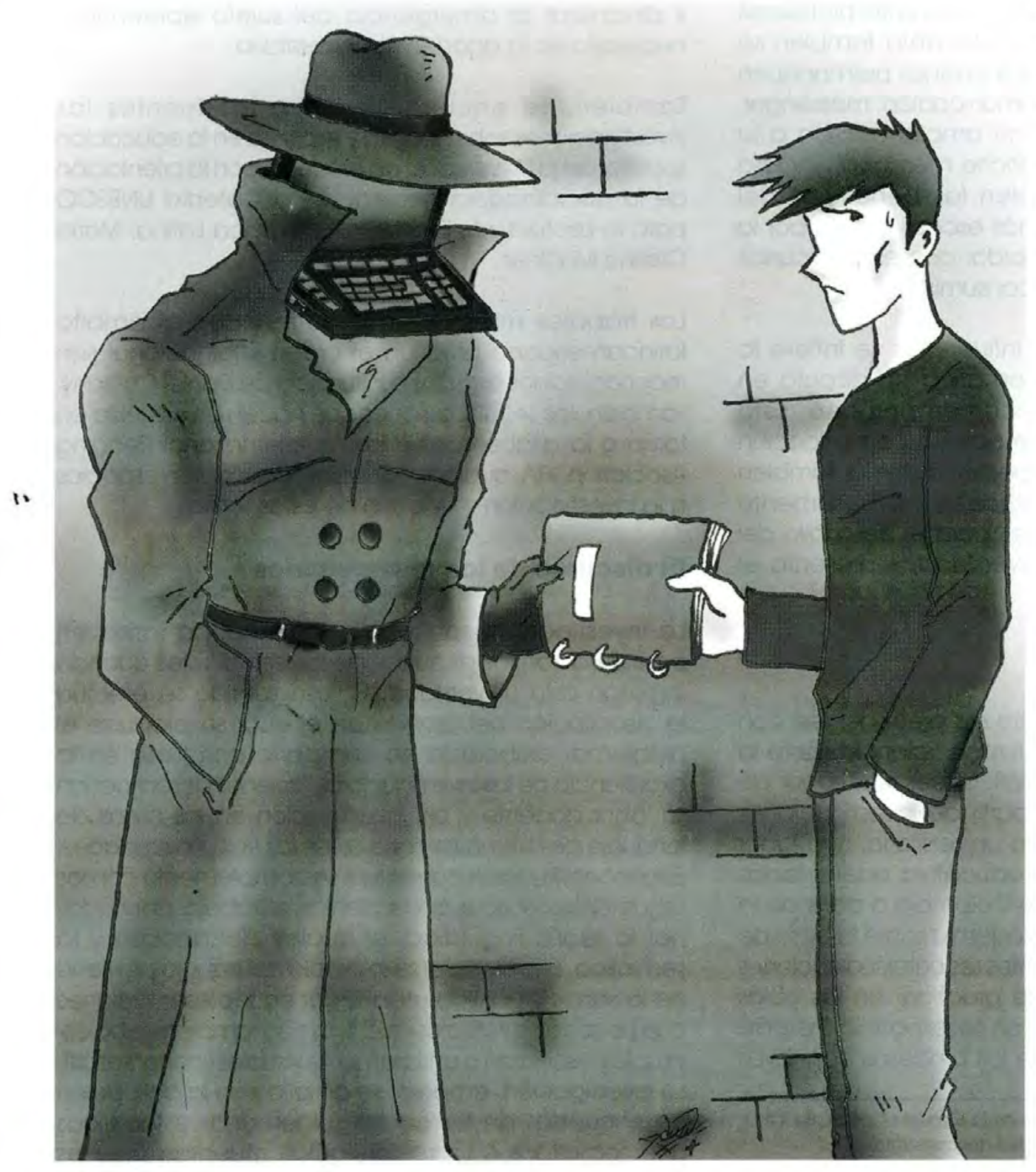

tendencias sobre la realidad de los semiohablantes que interactúan en la educación superior.

Además, el proyecto indaga la neogramática del estudiante universitario, considerada a partir de los múltiples intertextos con los cuales los dicentes están en contacto durante el transcurso de su educación básica y en los que se apoyan para seguir su proceso formativo.

La encuesta fue aplicada en UNIMINUTO Corporación Universitaria y la Universidad Colegio Mayor de Cundinamarca, en los primeros semestres de todas sus carreras. El documento constituido como resultado, en su estructura general, ha conformado un texto cuyo primer capítulo da a conocer el tema, los objetivos y la justificación reiterando la importancia del proyecto. 


\section{Descripción de las universidades}

Corporación Universitaria Minuto de Dios, UNIMINUTO Institución de educación superior de carácter privado. fundada por el Padre Rafael García Herreros, cuyo proyecto se orienta hacia los estratos sociales cuyas posibilidades económicas los marginan de la educación. Está regida por el principio de educación para todos por lo que prácticamente no realiza alguna clase de selección entre los postulantes que son admitidos con amplitud e interés en especial si sus dificultades son de orden económico. Su sede principal está en el Barrio Minuto de Dios, en el occidente de Bogotá, reúne 6.800 estudiantes.

Universidad Colegio Mayor de Cundinamarca. Institución de educación superior pública del Departamento de Cundinamarca con numerosos estudiantes favorecidos con carreras de formación media, como secretariado, que permiten ingresar de manera rápida al campo laboral. Su sede en la carrera $5^{a}$ de la capital colombiana recibe 6.000 estudiantes.

\section{Encuesta}

Aplicación. Una vez elaborada la encuesta ésta se aplicó a grupos de estudiantes seleccionados al azar, pertenecientes al primer semestre de todas las carreras de la Universidad Colegio Mayor de Cundinamarca y la Corporación Universitaria Minuto de Dios. En su elaboración se incluyeron preguntas orientadas a conocer las características de los estudiantes y a obtener tendencias en cuanto al contacto que mantienen los estudiantes con las nuevas tecnologías de la información y la clase de apropiación que hacen de sus recursos.

De esta encuesta se seleccionaron algunos ítemes que se ofrecen como adelanto del documento final, en elaboración. En particular, el segundo ítem del cuestionario Indaga sobre la disponibilidad de recursos en el hogar de los estudiantes, para indagar sobre posibles contactos comunicativos.

\section{Interpretación}

P. En el lugar donde habita dispone de televisor, videocasetera, video-juegos, computador, conexión a Internet, máquina de escribir.

\begin{tabular}{|l|l|l|l|}
\hline 1.En el lugar donde habita hay & U.C.M.C & UNIMINUTO & TOTAL \\
\hline Televisor & 184 & 209 & 393 \\
\hline Video-casetera & 102 & 124 & 226 \\
\hline Video-juegos & 64 & 94 & 158 \\
\hline Computador & 113 & 137 & 250 \\
\hline Conexión a Internet & 38 & 77 & 115 \\
\hline Máquina de escribir & 92 & 92 & 184 \\
\hline No sabe/no responde & 0 & 0 & 0 \\
\hline
\end{tabular}

En usta tabia se aprecia que los astudiartes encusstados, en el lugar oonde habian thanen, $29 \%$ televisor, $19 \%$ computacoon $17 \%$ video -casetera, $14 \%$ máquina de eseriba, $12 \%$ videnjuegos y solo un $9 \%$ restarite diapone de conexion a internet. Estos resultados permiter Intentr que determirados sectores sociales toda
que consituye basicamente un entretenimiento.

De acuerdo con estos resultados, aún el televisor aporta la conexión más numerosa, seguido de la videocasetera y los video juegos, encontrándose el computador en tercer lugar y la conexión a Internet a la zaga. De acuerdo con las diversas investigaciones sobre la televisión, se ha reconocido en ella las funciones informativa y persuasiva como fundamentales en el contacto con la audiencia, desde las cuales inciden en la formación de consumidores de entretenimiento $y$ de objetos.

En cuanto a la actualización tecnología de los hogares de los estudiantes que ingresan en estas universidades, se advierte que ella depende de la estratificación social y que los sectores altos son los que acceden con fluidez a estos recursos y los sectores medios y bajos lo hacen con más lentitud, dependiendo en muchos casos 
de que estos recursos estén presentes en las mismas instituciones o en las bibliotecas que conforman la red urbana.

El tercer item indaga sobre las actividades realizadas, relacionadas con el estudio como leer pero también con el extendido contacto con medios y entretenimientos electrónicos.

P. ¿Cuál de estas actividades realiza con mayor frecuencia?

\begin{tabular}{|c|c|c|c|}
\hline $\begin{array}{l}\text { 2. ¿Cuál de estas actividades } \\
\text { realiza con mayor frecuencia? }\end{array}$ & U.C.M.C & UNIMINUTO & TOTAL \\
\hline Leer & 95 & 110 & 205 \\
\hline Escribir & 71 & 73 & 144 \\
\hline Mirar televisión & 106 & 112 & 218 \\
\hline Chatear & 42 & 41 & 83 \\
\hline Navegar en Internet & 72 & 90 & 162 \\
\hline Jugar con video-juegos & 26 & 28 & 54 \\
\hline Escuchar radio & 134 & 142 & 276 \\
\hline Esludiar & 108 & 131 & 239 \\
\hline No sabe/no responde & 0 & 0 & 0 \\
\hline
\end{tabular}
4\% juega con viderojuegor.

Estos resultados resultan alentadores, aunque hay que tomar en cuenta que los estudiantes al ser encuestados pueden estar concientes de su papel como universitarios y las respuestas pudieran sesgarse, aunque es posible validar el imaginario que ubica a la lectura en los primeros lugares. En coherencia con la disponibilidad de conexión con las tecnologías de la información, que se indagaba en el ítem anterlor, radio y televisión ocupan el mayor tiempo de las actividades realizadas por los jóvenes; además, si se suman los resultados de leer y estudiar, estas actividades quedan claramente definidas por encima del entretenimiento, de las comunicaciones como chatear o de informarse como oír radio, En cuanto a navegar en Internet, además de la interactividad y la comunicación parte de esta navegación podría estar relacionada con la búsqueda de temas y contenidos formativos.

P. ¿Dónde realiza esta actividad (descritas en la tabla anterior)?

\begin{tabular}{|l|l|l|l|}
\hline $\begin{array}{l}\text { 3. ¿Dónde realiza esta } \\
\text { actividad? }\end{array}$ & U.C.M.C & UNIMINUTO & TOTAL \\
\hline En mi casa & 176 & 197 & 373 \\
\hline En casa de otras personas & 27 & 22 & 49 \\
\hline En mi lugar de trabajo & 37 & 45 & 82 \\
\hline En una institución educativa & 66 & 67 & 133 \\
\hline En un ciber-café & 43 & 51 & 94 \\
\hline No sabe/no responde & 0 & 0 & 0 \\
\hline
\end{tabular}

Es esta tabla se observa que los estudiantes encuestados realizan flicha actividad, $51 \%$ en su casa, otras personas.

Según estos resultados las actividades son realizadas mayoritariamente en la casa, probablemente sin disponer de recursos bibliográficos suficientes, con más televisores que computadores conectados por la red $y$, por lo tanto, con más posibilidades de que las actividades sean del orden del entretenimiento y de las comunicaciones (socialización de las emociones como ocurre en el chat) que de estudio. En cuanto al lugar de trabajo, es importante puesto que muchos de los encuestados son estudiantes nocturnos que trabajan durante el día, por lo cual el computador conectado en sus lugares de labor constituye un aporte al desarrollo de la formación universitaria y a la apropiación tecnológica que es lo que se dificulta en la instifución educativa. 
P. ¿Qué ve con frecuencia en televisión?

\begin{tabular}{|l|l|l|l|}
\hline $\begin{array}{l}\text { 4. ¿Qué ve con frecuencia en } \\
\text { televisión? }\end{array}$ & U.C.M.C & UNIMINUTO & TOTAL \\
\hline Noticieros & 110 & 125 & 235 \\
\hline Telenovelas o seriados & 68 & 68 & 136 \\
\hline Culturales o cientificos & 57 & 75 & 132 \\
\hline Cine & 84 & 108 & 192 \\
\hline Realities & 38 & 30 & 68 \\
\hline Deportes & 53 & 71 & 124 \\
\hline Musicales y variedades & 81 & 109 & 190 \\
\hline Religiosos & 3 & 11 & 14 \\
\hline No sabe/no responde & 2 & 0 & 2 \\
\hline
\end{tabular}

En esta tabla se observa que los estudiantes emcuestados ven en felevidion, $23 \%$ noticieros, $18 \%$ ciné, $17 \%$ musica y variedades. $12 \%$ culturales o cientificos, $12 \%$ tolenovelas. $11 \%$ deportes, $8 \%$ realities $y$ of $1 \%$ retigiosos

Los noticieros ocupan el primer lugar; sin embargo, el resultado no se interpreta como es debido sin el conocimiento de las características de estos formatos mediáticos. En la emisión colombiana, los que son destacados por el denominado rating (confirmado por esta encuesta) dedican gran parte de su tiempo a informaciones deportivas subjetivamente tratadas por los comentaristas y a la franja light en uso de un eufemismo que signifique que no se trata de la noticia periodística sino de entretenimiento; además, debe tomarse en cuenta la franja publlicitaria y la autopromoción del emisor. El resto de la programación podría denominarse con el término que muchos de sus usuarios usan cuando hacen referencia a estos programas: telebasura.

Frente a la televisión que aparece como una presencia heredada por los jóvenes de su grupo familiar y social aparecen el computador e Internet en la renovación del contexto mediatizado.
P. ¿Cuánto tiempo, por semana, dedica al correo electrónico?

\begin{tabular}{|c|c|c|c|}
\hline $\begin{array}{l}\text { 5. ¿Cuánto tiempo, por semana, } \\
\text { dedica al correo electrónico? }\end{array}$ & U.C.M.C & UNIMINUTO & TOTAL \\
\hline Ninguno & 10 & 7 & 17 \\
\hline Menos de 3 horas & 120 & 129 & 249 \\
\hline 3 a 6 horas & 43 & 50 & 93 \\
\hline 6 a 9 horas & 7 & 11 & 18 \\
\hline 90 más & 10 & 16 & 26 \\
\hline No sabe/no responde & 0 & 0 & 0 \\
\hline
\end{tabular}

Aquí se constata la dedicación en un gran número de horas al correo electrónico, que exige habilidades expresivas y comunicativas muy favorables para la vida académica.

P. ¿Cuánto tiempo, por semana, se dedica a chatear?

\begin{tabular}{|c|c|c|c|}
\hline $\begin{array}{l}\text { 6. ¿Cuánto tiempo, por semana, se } \\
\text { dedica a chatear? }\end{array}$ & U.C.M.C & UNIMINUTO & TOTAL \\
\hline Ninguno & 96 & \begin{tabular}{|l|}
96 \\
\end{tabular} & 192 \\
\hline Menos de 3 horas & 69 & 82 & 151 \\
\hline 3 a 6 horas & 17 & 25 & 42 \\
\hline 6 a 9 horas & 2 & 7 & 9 \\
\hline 9 ó más & 6 & 3 & 9 \\
\hline No sabe/no responde & 0 & 0 & 0 \\
\hline
\end{tabular}

En esta tobla se aprecis que los estudiantes encuestados, por semane
menos do 3 horas. $10 \% 3$ a 5 horas. $2 \%, 6$ a 9 horas y $2 \% 90$ mis horas.

El chat simboliza, incluso más que internet, el contexto contemporáneo. Es el nuevo espacio de los jóvenes, como antes pudieron serlo los centros urbanos, en el cual invierten mucho tiempo y donde se conectan con sus pares, personas con quienes comparten intereses los cuales, se infiere, no siempre estarán relacionados con su desarrollo cognoscitivo. Aunque, la población encuestada aún no ha ingresado en su totalidad a esta actividad, a la cual sólo 9 personas le dedican más de 9 horas de su cotidianidad. En este sentido, cabe preguntarse ¿cuándo asisten a los cursos, leen y estudian? y ¿cuánto tiempo dedican a la vida académica? 
P. ¿Cuando está en Intemet busca, preferencialmente?

\begin{tabular}{|c|c|c|c|}
\hline $\begin{array}{l}\text { 7. Cuando está en Internet } \\
\text { busca, preferencialmente }\end{array}$ & U.C.M.C & UNIMINUTO & TOTAL \\
\hline Publicidad & 24 & 37 & 61 \\
\hline $\begin{array}{lll}\text { Información } \\
\text { enciclopédica }\end{array}$ & 114 & 109 & 223 \\
\hline Música y variedades & 96 & 121 & 217 \\
\hline $\begin{array}{l}\text { Literatura relacionada con la } \\
\text { profesión }\end{array}$ & 40 & 62 & 102 \\
\hline Periódicos y revistas & 25 & 45 & 70 \\
\hline Articulos de autoayuda & 25 & 41 & 66 \\
\hline Articulos científicos & 43 & 52 & 95 \\
\hline No sabe/no responde & 1 & 0 & 1 \\
\hline
\end{tabular}

En la gratica antenior se aprecia que de los estudantes encuestados buscarn en Internet. $28 \%$

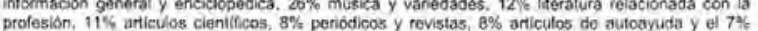
profesion,

Al igual que en el ítem que indaga sobre las actividades de los jóvenes, en este caso salen favorecidas actividades de apoyo para la vida estudiantil, según

n. puede verse en los resultados de la información general y enciclopédica. También, es interesante constatar como entre ambas universidades existen más bien coincidencias que diferenciaciones.

P: Internet sirve para:

\begin{tabular}{|l|l|l|l|}
\hline \multicolumn{1}{|c|}{ 8. Internet le sirve para } & $\begin{array}{c}\text { U.C.M. } \\
\text { C }\end{array}$ & $\begin{array}{c}\text { UNIMINUT } \\
\text { O }\end{array}$ & $\begin{array}{c}\text { TOTA } \\
\text { L }\end{array}$ \\
\hline Resolver trabajos de estudio & 181 & 190 & 371 \\
\hline Solucionar aspectos laborales & 39 & 39 & 78 \\
\hline $\begin{array}{l}\text { Localizar articulos de superación } \\
\text { personal }\end{array}$ & 14 & 19 & 33 \\
\hline Leer las noticias & 49 & 71 & 120 \\
\hline $\begin{array}{l}\text { Encontrar diversión, distracción y yetrenimiento } \\
\text { entretende }\end{array}$ & 111 & 117 & 228 \\
\hline No sabe/no responde & 2 & 0 & 2 \\
\hline
\end{tabular}

En esta tablo 5 ap aprecia que los estudianies oncuestados consiceran que internel sirvo para. $46 \%$ Resolver

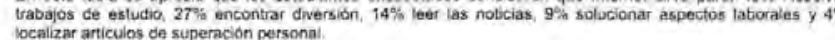

El resultado de Internet como apoyo y parte de la vida estudiantil queda confirmado con estos resultados. La tarea se soluciona con Internet, aún como plagio cuando se baja un ensayo sin que ni siquiera se lea, cuando se corta y se pega, prácticas muy difundidas entre los jóvenes usuarios de la red.
Para reconocer otras influencias mediáticas como el cine, el cual es una clase de influencia que después de haber formado parte de las comunicaciones masivas está reservado, en la actualidad, para ciertas élites del mismo modo que los libros. Realizamos un paralelismo que aporta ideas sobre la manera como el contacto se modifica vertiginosamente dando lugar a la contemporaneidad.

\section{P. ¿Cuánto tiempo, por semana, dedica al cine?}

\begin{tabular}{|l|l|l|l|}
\hline \multicolumn{1}{|c|}{$\begin{array}{c}\text { 9. ¿Cuánto tiempo, por semana, } \\
\text { dedica al cine? }\end{array}$} & U.C.M.C & UNIMINUTO & TOTAL \\
\hline Ninguno & 75 & 66 & 141 \\
\hline menos de 3 horas & 88 & 102 & 190 \\
\hline 3 a 6 horas & 21 & 30 & 51 \\
\hline 6 a 9 horas & 6 & 11 & 17 \\
\hline 9 ó más & 1 & 1 & 2 \\
\hline No sabe/no responde & 2 & 0 & 2 \\
\hline
\end{tabular}

En esta laba se puede abservar que las estudantes encuestados decican por semana, al

Los jóvenes estudiantes mantienen un contacto reducido con el cine. Además, probablemente incluyen en sus respuestas películas que encuentra en la programación de la televisión, sin que se trate de la elección que lleva hasta la sala de exhibición. El siguiente ítem ayuda a reafirmar este supuesto.

\section{P. ¿Qué tipos de películas ve?}

\begin{tabular}{|c|c|c|c|}
\hline $\begin{array}{l}\text { 10. ¿Qué tipos de } \\
\text { peliculas ve? }\end{array}$ & U.C.M.C & UNIMINUTO & TOTAL \\
\hline Drama & 76 & 77 & 153 \\
\hline Terror (misterio) & 100 & 111 & 211 \\
\hline Animadas & 62 & 66 & 128 \\
\hline Humoristicas & 84 & 91 & 175 \\
\hline Ciencia ficción & 79 & 82 & 161 \\
\hline Acción & 88 & 83 & 171 \\
\hline Documentales & 59 & 89 & 148 \\
\hline Históricas & 45 & 69 & 114 \\
\hline No sabe/no responde & 10 & 0 & 10 \\
\hline
\end{tabular}

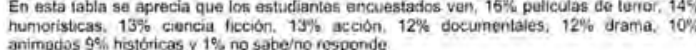


Se trata fundamentalmente de filmes comerciales, de dudosa calidad, que son los que suele ofrecer la programación de la TV. La tendencia al entretenimiento, caracteristica de las culturas juveniles y estudiantiles, se establece con los siguientes ítemes.

P.: ¿Cuánto tiempo pasa, por semana en lugares de baile?

\begin{tabular}{|l|l|l|l|}
\hline $\begin{array}{c}\text { 11. ¿Cuánto tiempo pasa, por } \\
\text { semana } \\
\text { en lugares de baile? }\end{array}$ & U.C.M.C & UNIMINUTO & TOTAL \\
\hline Ninguno & 44 & 71 & 115 \\
\hline menos de 3 horas & 83 & 64 & 147 \\
\hline 3 a 6 horas & 40 & 52 & 92 \\
\hline 6 a 9 horas & 14 & 15 & 29 \\
\hline 9 o mas & 11 & 4 & 15 \\
\hline No sabe/no responde & 2 & 0 & 2 \\
\hline
\end{tabular}

En esta table sé observiva que los eshudiantes encuéstadós pasan por semana, en lugares de baile

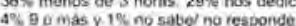

Los resultados muestran que la mayoria de los estudiantes concurre a bailes. SI se suman las cifras de menos de 3 horas y 3 a 6 horas indica que hay muchos más estudiantes que además de ver televisión, leer, ingresar a la red para extraer información, leer y escribir correos electrónicos y chatear también le dedican tiempo a otras actividades lúdicas y deportivas. La suma total de actividades amenaza con exceder las veinticuatro horas diarias.

A pesar de ello, la lectura puede verse favorecida debido a que se la privilegia idealmente.

P. ¿Cuántas veces por semana compra el periódico?

\begin{tabular}{|c|c|c|c|}
\hline $\begin{array}{l}\text { 12. ¿Cuántas veces por semana compra } \\
\text { el periódico? }\end{array}$ & U.C.M.C & UNIMINUTO & TOTAL \\
\hline Nunca & \begin{tabular}{|l|}
87 \\
\end{tabular} & 82 & 169 \\
\hline Todos los dias & 15 & 23 & 38 \\
\hline Una vez por semana & 64 & 74 & 138 \\
\hline Dos veces por semana & 14 & 19 & 33 \\
\hline Entre 3 y 5 veces por semana & 8 & 9 & 17 \\
\hline No sabe/no responde & 2 & 0 & 2 \\
\hline
\end{tabular}

En esta tibla so observa que 105 estudiantios ancuestados compran el perbobico, por semana. $42 \%$ no adquieren dicho medio, $35 \%$ una vez por sernana, $10 \%$ lodos las dias, BS dos veces por semana, $4 \%$ entre 3 y 5 veces
por semana y $1 \%$ no sabeino responde.
Hay que considerar que los periódicos se encuentran en la red y que, sumado a otras razones, el medio impreso casi nunca se compra en los hogares de los jóvenes estudiantes. Además, al igual que todos los medios también puede constituirse fundamentalmente en entretenimiento más que en fuente de información y formación. Por tanto, se agrega otro punto.

\section{P. ¿Qué lee en el periódico?}

\begin{tabular}{|l|l|l|l|}
\hline \multicolumn{1}{|c|}{$\begin{array}{c}\text { 13. ¿Qué lee en el } \\
\text { periódico? }\end{array}$} & U.C.M.C & UNIMINUTO & TOTAL \\
\hline Editorial & 36 & 46 & 82 \\
\hline Economia & 57 & 63 & 120 \\
\hline Política & 34 & 66 & 100 \\
\hline Espectáculo & 53 & 52 & 105 \\
\hline Clasificados & 42 & 49 & 91 \\
\hline Tiras cómicas & 48 & 47 & 95 \\
\hline Cultura & 107 & 124 & 231 \\
\hline Clencia & 76 & 90 & 166 \\
\hline No sabe/no responde & 23 & 0 & 23 \\
\hline
\end{tabular}

En esta labla se aprecia que los estudiantes encuestados leart on ef periodico. $24 \%$ cuifturas A\% editanal y $2 \%$ no babeing responde

Los resultados son muy interesantes, a pesar de que los periódicos que leen no traen casi información científica ni cultural: sin embargo, resulta de interés para los estudiantes. Aunque las cifras de alguna manera estén influenciadas por la conciencia de los encuestados, sin embargo, el hecho de que se privilegien estos aspectos resulta positivo para esta indagación.

\section{Conclusiones}

A partir de la aplicación de la encuesta y de la lectura e interpretación de los resultados, surge que la televisión es el medio masivo que la gran mayoría de estudiantes tiene en sus hogares, lo cual lleva a suponer una influencia de la programación en la audiencia; pero, contrario a esto se descubre que la actividad más realizada por los dicentes es escuchar 
radio, especialmente en sus hogares; es decir, que aún se prefiere acudir a un medio más cercano a la comunicación oral.

Además, el tiempo que actualmente dedican los estudiantes a ver televisión es bajo, debido a las múltiples ocupaciones que enfrentan diariamente; ejemplo de ello son los alumnos que trabajan en el dia y acuden a clases en la noche, pero lo más sorprendente es afirman que dicho tiempo lo dedican a ver noticieros, sin duda, esto se debe a que la realidad del país ha llevado a los lóvenes a conclenciarse sobre su situación y madurar en el sentido que es fundamental mantenerse al tanto de lo que sucede en el entorno, ya que esto los hace más competentes.

De otra manera, al tratar el tema de Internet, el cual es " por estos días uno de los medios más conocidos, ya sea por la facilidad de acceder a él o su rapidez para informar, se aprecia que los encuestados acuden a la web, principaimente, para resolver trabajos de estudio y buscar información general y enciclopédica; pues actualmente es raro encontrar a un alumno buscando sus tareas en libros, frente a cualquier pregunta, por fácil que sea, lo primero es buscar en el ciberespacio, incluso ya se están acabando los libros de recortes porque todas las imágenes se bajan del computador.

Luego, al preguntar por el correo electrónico se observa que a pesar del auge de este medio de comunicación. el tiempo que dedican por semana es bajo, pues en su mayoria respondieron menos de tres horas, aunque es totalmente comprensible debido a que la información que circula por esta vía son las famosas cadenas basura y pomografia, entre otros.

Igualmente, al cuestionar sobre el chat se observa que aún parece no ser relevante para los alumnos, probablemente por lo vacio de las conversaciones o la impersonalidad que se maneja, aunque hay casos en que se llegan a consolidar fuertes relaciones a través de él y, se puede inferir, que su uso va en aumento.

Por otro lado, pese al incremento de la piratería, el cine aún logra seducir los sentidos y preferencias de los encuestados, al generar gran cantidad de sentimientos que no se basan, como el caso de la televisión, en atraer al público con historias sobre las pandillas de Medellin o realities sobre sobrepeso, ofertados por la programación comercial.

Para cerrar, se ofrece un orden que establece las prioridades en la comunicación y contactos con libros, con medios Y TIC, de los ingresantes a la universidad. En relación con ello, se establecen las actitudes y hábitos relacionados con la adquisición de:

\section{Recomendaciones}

Se sugiere que el estudio sobre la neogramática en el estudiante universitario, sea dífundido en el ámbito universitario, para que coadyuve en la formación académica y de investigación en la educación superior.

La universidad como eje del saber debe tener en cuenta dentro de su estructura curicular elementos teóricos, metodológicosy prácticos que lleven al educando a interesarse por la lectura, escritura y el análisis de textos.

Implementar en las universidades programas de lectura encaminados a profundizar el área de estudio de cada educando según su carrera.

Concebir programas radiales y televisivos que generen en los estudiantes hábitos de lectura, aprovechamiento del tiempo libre y uso apropiado del lenguaje, en beneficio de su formación profesional y personal.2? 


\section{Bibliografía}

BETTELHEIM, Bruno y ZELAM, Karen, Aprender a leer, Barcelona, Grijalbo, 1983.

CASCO, Miriam, "Los problemas del léxico en las producciones escritas de estudiantes universitarios", ponencia al VII Congreso Nacional de la SAL, Neuquén, 1998.

CASCO, Miriam, "Competencias lectoras de los ingresantes a la Universidad: los problemas del procesamiento de información", Segundo Coloquio Latinoamericano de Analistas del Discurso, organizado por Asociación Latinoamericana de Estudios del Discurso, Universidad de la Plata y Universidad de Buenos Aires, La Plata-Buenos Aires, 25 al 29 de agosto de 1997.

CASSANY, Daniel, LUNA, Marta y SANZ, Gloria, Ensen̂ar lengua, Barcelona, 1994.

DUBOIS, Jean y otros, Diccionario de lingüística, Madrid, Allanza, 1979.

ECO, Umberto, Lector in fábula, Barcelona, Lumen, 1989.

FERREIRO, Emilia y GÓMEZ, Margarita (comp.), Nuevas perspectivas sobre los procesos de lectura y escritura, México, Siglo Veintiuno, 1982 ,

GARCÍA PÉREZ, Jorge, Estrategias lectoras e idiomáticas, Medellin, Lealón, 1995.

GIRÓN, María Stella y VALLEJO, Marco Antonio, Producción e interpretación textual, Medellín, Editorial Universidad de Antioquia, 1992.

GOODMAN, Kenneth, "El proceso de lectura: consideraciones a través de las lenguas y del desarrollo". en Nuevas perspectivas sobre los procesos de lectura y escritura, México, Siglo Veintiuno,págs. 13-28, 1982.

GREIMAS, J., Del Sentido II، Ensayos semióticos، Madrid. Editorial Gredos, 1989.

IRWIN, Judith y DOYLE, Mary Anne (comp.), Conexiones entre lectura y escritura, Buenos Aires, Aique, 1992.

JURADO VALENCIA, Fabio, "La lectura: los movimientos interpretativos son movimientos evaluativos", en Entre la lectura y la escritura, Santa fe de Bogotá, Magisterio, Mesa Redonda, 1997.

KLINKENBERG, Jean-Marie, Manual se semiótica general, Bogotá, Universidad Jorge Tadeo Lozano, 2005.

MARTÍNEZ, Betty, MUÑOZ, Clarena, y ASQUETA, Maria Cristina, Érase una vez, Análisis crítico de la telenovela, Bogotá, Corporación Universitaria Minuto de Dios, 2006.

MARTINEZ, Maria Cristina (comp.), Los procesos de la lectura y la escritura, Santiago de Cali, Editorial Universidad del Valle, 1997.

MOLINER, María, Diccionario de uso del español, Madrid, Credos, vol., 21981.

ONG, Walter, Oralidad y Escritura, Tecnologías de la palabra, México, Fondo de Cultura Económica, 1987. PARDO ABRIL, Neyla Graciela, "Las superestructuras textuales en los procesos pedagógicos", en Forma y Función (6). Bogotá, abril de 1992, págs. 9 - 23.

REAL ACADEMIA ESPAÑOLA, Diccionario de la Lengua Española, Tomos I y II, Vigésima primera edición, Madrid, Espasa Calpe, 1992.

SAUSSURE, Ferdinad de, Curso de lingüistica general, Editorial Losada, Buenos Aires, 1945.

DIJK, Teun van, Discurso y dominación, Bogotá, Universidad Nacional de Colombia, 2004.

1978. La ciencia del texto, Barcelona, Paidós, Estructuras y funciones del discurso, México, Siglo Veintiuno, 1980. Texto y contexto, Madrid, Cátedra, 1995.

ZUBIRIA SAMPER, Miguel de, Teoría de las seis lecturas, Tomo I y ll, Santa fe de Bogotá, Fondo de Publicaciones Bernardo Herrera Merino, 1995.

ZULETA, Estanisiao, "Conferencia sobre la lectura", en Los procesos de la lectur, Santa fe de Bogotá, Magisterio, Mesa Redonda 30, 1995, págs. 11 - 37. 